\title{
Avaliação de painéis aglomerados de Toona ciliata produzidos com diferentes densidades e teores de resina
}

\section{Evaluation of particleboard of Toona ciliata produced with different densities and resin levels}

\author{
Rosilani Trianoski', Setsuo Iwakiri' e Jorge Luis Matos'
}

\begin{abstract}
1 Departamento de Engenharia e Tecnologia Florestal, Universidade Federal do Paraná - UFPR, Curitiba, PR, Brasil.rosilani@ufpr.br; setsuo@ufpr.br; jmatos@ufpr.br
\end{abstract}

\section{RESUMO}

Este trabalho teve como objetivo avaliar as propriedades físicas e mecânicas de painéis aglomerados de Toona ciliata produzidos com diferentes densidades e teores de resina. Foram utilizadas árvores provenientes de um plantio experimental localizado em Corupá-SC. As árvores coletadas foram seccionadas em toras e amostras de todas as posições longitudinais foram utilizadas para a determinação das propriedades da madeira e para a geração das partículas. A avaliação das propriedades da madeira consistiu na determinação da densidade básica e das propriedades químicas, as quais seguiram os procedimentos das normas COPANT e TAPPI, respectivamente. Os painéis foram produzidos segundo um delineamento inteiramente casualizado, utilizando densidades de $0,60 \mathrm{~g} / \mathrm{cm}^{3} ; 0,70 \mathrm{~g} / \mathrm{cm}^{3} \mathrm{e} 0,80 \mathrm{~g} / \mathrm{cm}^{3}$, e, $6 \%$ e $8 \%$ de resina, e prensados à temperatura de $160^{\circ} \mathrm{C}$, pressão específica de $4 \mathrm{MPa}$ e tempo de 8 minutos. As propriedades físicas e mecânicas foram avaliadas de acordo com a metodologia proposta pela Norma Européia e pela Norma Brasileira, sendo efetuados os ensaios de densidade aparente (CEN, 2002d), absorção de água e inchamento em espessura (CEN, 2002b), flexão estática (CEN, 2002a), tração perpendicular (CEN, 2002c) e resistência ao arrancamento de parafuso (ABNT, 2006b). Os resultados indicaram que todos os tratamentos apresentaram os requisitos mínimos estabelecidos pelas normas de referência. Os painéis produzidos com menor quantidade de matéria prima $\left(0,60 \mathrm{~g} / \mathrm{cm}^{3} \mathrm{e} 6 \%\right)$, além de atenderem os requisitos normativos, indicaram uma economia de até $23,73 \%$ na quantidade de partículas e até $42,80 \%$ nas quantidades de resina e catalisador, quando comparado com os demais tratamentos.

PALAVRAS CHAVE: Cedro australiano, densificação, espécies alternativas, redução de matéria prima, uréia-formaldeído.

\section{ABSTRACT}

This study aimed to evaluate the physical and mechanical properties of particleboard produced of Toona ciliata with different densities and resin levels. Trees from an experimental plantation located in Corupá-SC were used. The trees were cut into logs collected and samples of all longitudinal positions were used to determine the properties of the wood and the generation of particles. Evaluation of wood properties consisted in determining the wood density and chemical properties, which followed the procedures of COPANT standards and TAPPI, respectively. The panels were produced according to a randomized design, using nominal density of $0,60 \mathrm{~g} / \mathrm{cm}^{3}, 0,70 \mathrm{~g} / \mathrm{cm}^{3}$ and $0,80 \mathrm{~g} / \mathrm{cm}^{3}$, and $6 \%$ and $8 \%$ resin and pressed at a temperature of $160^{\circ} \mathrm{C}$, at an specific pressure of $4 \mathrm{MPa}$ and in atime of 8 minutes. The physical and mechanical properties were evaluated according to the methodology proposed by the European Standard and the Brazilian Standard, the tests made being apparent density (CEN, 2002d), water absorption and thickness swelling (CEN, 2002b), static bending (CEN, 2002a), perpendicular traction (tension) (CEN, 2002c) and screw pullout resistance (ABNT, 2006b). The results indicated that all treatments coplied with the minimum requirements established by the reference standards. The panels produced with fewer raw materials (density of $0,60 \mathrm{~g} / \mathrm{cm}^{3}$ and $6 \%$ resin), in addition to meeting the regulatory requirement, indicated a savings of up to $23,73 \%$ on the amount of particles and up to $42,80 \%$ on the amounts of resin and catalyst, when compared with other treatments.

KEYWORDS: Autralian cedar, densification, species alternatives, reduction of the raw material, urea-formaldehyde. 


\section{INTRODUÇÃO}

A indústria de painéis aglomerados contribui ativamente com o desenvolvimento econômico e tecnológico em função da ampla variedade e grande quantidade de produtos gerados. Segundo Tomaselli (1999) entre as décadas de 70 e 90, a produção mundial de painéis aglomerados teve aumento expressivo, passando de 30 milhões de metros cúbicos em 1975, para mais de 60 milhões de metros cúbicos em 1995. De acordo com a Abipa (2012), a capacidade nominal da indústria de painéis aglomerados no Brasil é de 4,79 milhões de metros cúbicos e a produção em 2011 foi de 3,07 milhões de metros cúbicos.

Esta elevada produção, assim como a projeção de aumento demonstrada por meio das informações da capacidade nominal instalada demonstra que o volume de madeira requerido pelo setor será ainda maior. Desta forma, para atender a demanda de matéria-prima cada vez mais crescente, torna-se necessário não somente aumentar as áreas de plantio com espécies atualmente utilizadas, mas também buscar outras espécies de rápido crescimento que possam contribuir de forma quantitativa e qualitativa para suprir as necessidades das indústrias (Trianoski, 2010).

Entre as diversas espécies de rápido crescimento destaca-se a Toona ciliata. É uma espécie nativa da Austrália (Keenan et al., 1997; Herwitz et al., 1998), pertencente a Família Meliaceae e popularmente conhecida como cedro australiano (Lamprecht, 1990).

No Brasil, esta espécie encontrou condições favoráveis ao seu crescimento e produção de madeira (Boletim Florestal, 2008), onde em Minas Gerais, plantios submetidos a técnicas adequadas de manejo chegam a atingir uma altura média de $20 \mathrm{~m}$ e DAP de $40 \mathrm{~cm}$ aos 12 anos de idade (Ziech, 2008).

Entre as várias utilizações descritas, como por exemplo, construção, lâminas e compensados decorativos (Paiva et al., 2007), portas, janelas, carrocerias, molduras, trabalhos de torneamento (Dordel y Simard, 2009), produção de móveis, instrumentos musicais e outras finalidades especiais (Lamprecht, 1990) esta espécie demonstrou-se adequada para a produção de painéis de madeira aglomerada (Sá, 2009; Trianoski, 2010).
Entre os inúmeros fatores que exercem influência sobre os painéis de madeira aglomerada destaca-se a densidade da madeira. Esta importante variável afeta tanto as propriedades do produto final como os parâmetros do processo (Kelly, 1977; Moslemi, 1974; Maloney, 1993). Segundo Iwakiri (2005) a produção de aglomerados requer baixa densidade, e Maloney (1993) complementa que espécies com até $0,55 \mathrm{~g} / \mathrm{cm}^{3}$ são as mais adequadas por atingirem uma razão de compactação entre 1,3 e 1,6; sendo esta faixa, a considerada ideal para o processo de densificação e consolidação do painel.

De acordo com as informações relatadas por Kelly (1977), espécies de menor densidade possibilitam a produção de painéis aglomerados com uma área de contato entre partículas satisfatória. Isto ocorre pelo fato de que espécies com densidade mais baixa necessitam de um maior volume de partículas para se obter um painel com uma mesma densidade nominal, e o conseqüente aumento do contato entre elas, resulta em um aumento expressivo de resistência (Brito, 1984). Moslemi (1974) complementa ainda, que painéis confeccionados com espécies de baixa densidade apresentam aumento na resistência à flexão, resistência a tração e módulo de elasticidade. Já a resistência ao arrancamento de parafuso, a absorção de água e o inchamento em espessura são pouco afetados.

Em relação a esta característica, a Toona ciliata produz madeira com densidade básica relativamente baixa, onde os resultados apresentados pela literatura são de $0,337 \mathrm{~g} / \mathrm{cm}^{3}$ (Pereyra et al., 2006), $0,245 \mathrm{~g} / \mathrm{cm}^{3}$ a $0,364 \mathrm{~g} /$ $\mathrm{cm}^{3}$ (Ziech, 2008), 0,320 g/ $\mathrm{cm}^{3}$ (Bufalino et al., 2012). Apesar das inúmeras vantagens apresentadas por esta característica, o baixo valor da densidade implica em uma desvantagem, a qual está relacionada ao elevado volume de madeira consumido no processo produtivo, já que a matéria prima é utilizada em termos de massa de partículas secas $(3 \%)$, e quanto menor a densidade, maior o volume de toras para compor um painel ou lote com uma determinada densidade nominal.

A baixa densidade da madeira implica ainda no consumo de resina e de outros aditivos. Espécies que apresentam baixa densidade necessitam de um maior volume de 
partículas para compor um painel de determinada densidade nominal, logo, a área superficial é aumentada, sendo necessária uma maior quantidade de resina (Marra, 1992) para se obter boa aderência entre as partículas e uma colagem satisfatória.

\section{OBJETIVOS}

Neste contexto, considerando a baixa densidade da Toona ciliata e suas possíveis implicações sobre o consumo de madeira e resina no processo produtivo, este trabalho tem como objetivo avaliar as propriedades físicas e mecânicas de painéis aglomerados produzidos a partir de madeiras desta espécie com diferentes densidades e diferentes percentuais de resina.

\section{MATERIAIS E MÉTODOS}

Para o desenvolvimento deste trabalho foram selecionadas 5 árvores de Toona ciliata, provenientes de um plantio experimental com 17,3 anos de idade localizado em Corupá - SC, a latitude de 26²3'19,32'O, longitude $49^{\circ} 16^{\prime} 50,74$ 's, e altitude de $75 \mathrm{~m}$.

As árvores selecionadas foram seccionadas de acordo com a altura comercial $(0 \%, 25 \%, 50 \%, 75 \%$ e $100 \%$ ), obtendo-se amostras para determinação da densidade básica e propriedades químicas, e toras para a geração das partículas. A densidade básica da madeira foi determinada de acordo com procedimentos recomendados pela Norma COPANT 461/1972 (COPANT, 1972). Já para a determinação das propriedades químicas as amostras foram transformadas em cavacos e posteriormente em serragem, a qual foi classificada de acordo com a Norma TAPPI 264 (TAPPI, 1997a). As análises químicas realizadas foram solubilidade em água fria e quente (TAPPI 207:1999; TAPPI, 1999a), extrativos em etanol tolueno (TAPPI 280:1999; TAPI, 1999b), extrativos totais (TAPPI 204:1997; TAPPI, 1997b) e materiais inorgânicos (TAPPI 211:2002; TAPPI, 2002a) e pH (TAPPI 252: 2002; TAPPI, 2002b).

Para a produção dos painéis, as toras foram novamente seccionadas, obtendo-se toretes de todas as posições longitudinais, a fim de se compreender toda a variabili- dade existente nas árvores. A partir do processamento dos toretes foram obtidos cavacos, e estes após terem sido submetidos a um moinho de martelo originaram partículas do tipo "sliver". As partículas foram classificadas em peneiras com granulometrias de 8 e 30 mesh. Após esta classificação, foi efetuada a secagem final obtendo-se partículas com um teor de umidade próximo a $3 \%$.

Os painéis foram produzidos de acordo com o delineamento experimental proposto na tabela 1 , com resina uréia-formaldeído (teor de sólidos de 65,02\%, viscosidade Brookfield de $650 \mathrm{cP}, \mathrm{pH}$ de 8,57 , densidade de $1,29 \mathrm{~g} / \mathrm{cm}^{3}$ e tempo de gelatinização de $1,06 \mathrm{~min}$ ) e, $1 \%$ de parafina. Foram manufaturados de forma homogênea com dimensões de $50 \mathrm{~cm} \times 50 \mathrm{~cm} \times 1,5 \mathrm{~cm}$, e prensados à temperatura de $160^{\circ} \mathrm{C}$, pressão específica de $4 \mathrm{MPa}$ e tempo de 8 minutos. O delineamento utilizado foi o inteiramente casualizado com três repetições (chapas) por tratamento.

TABEla 1. Delineamento Experimental.

\begin{tabular}{cl}
\hline Tratamento & Composição \\
\hline 1 & Densidade nominal 0,80 - Teor de resina 8\% \\
\hline 2 & Densidade nominal 0,70 - Teor de resina 8\% \\
\hline 3 & Densidade nominal 0,60 - Teor de resina 8\% \\
\hline 4 & Densidade nominal 0,80 - Teor de resina 6\% \\
\hline 5 & Densidade nominal 0,70 - Teor de resina 6\% \\
\hline 6 & Densidade nominal 0,60 - Teor de resina 6\%
\end{tabular}

Após a manufatura, os painéis foram conduzidos à câmara de climatização com condições ambientais controladas $\left(20{ }^{\circ} \mathrm{C} \pm 2{ }^{\circ} \mathrm{C}\right.$ e $65 \% \pm 5 \%$ UR), até atingirem umidade de equilíbrio.

As propriedades físicas e mecânicas dos painéis foram avaliadas de acordo com a metodologia proposta pela Norma Européia e pela Norma Brasileira. A avaliação das propriedades físicas consistiu na determinação da Densidade aparente (CEN, 2002d) e da Absorção de Água e Inchamento em Espessura após 2 e 24 horas (CEN, 2002b). Já a determinação das propriedades mecânicas consistiu 
no ensaio de Flexão Estática (CEN, 2002a), Tração Perpendicular à Superfície (CEN, 2002c) e Resistência ao Arrancamento de Parafuso (ABNT, 2006b). Os valores experimentais foram comparados com os requisitos propostos pela Norma EN 312:2003 e NBR 14810-2:2006 (ABNT, 2006a).

Os dados obtidos foram submetidos à análise estatística (95\% de probabilidade) por meio dos testes de Grubbs, Shapiro Wilks, Bartlett, Análise de Variância,e Comparação de Médias de Tukey.

\section{RESULTADOS E DISCUSSÃO}

\section{Propriedades da madeira}

Os valores médios obtidos para as propriedades físicas e químicas da madeira de Toona ciliata são apresentados na tabela 2 .

TABEla 2. Resultados médios das propriedades físicas e químicas da madeira de Toona ciliata.

\begin{tabular}{lcc}
\hline \multicolumn{1}{c}{ Propriedade } & $\begin{array}{c}\text { Valor } \\
\text { médio }\end{array}$ & $\begin{array}{c}\text { Coeficiente } \\
\text { de Variação } \\
\text { (\%) }\end{array}$ \\
\hline Densidade básica $\left(\mathrm{g} / \mathrm{cm}^{3}\right)^{*}$ & 0,33 & 10,42 \\
\hline Solubilidade em água fria (\%) & 6,64 & 4,03 \\
\hline Solubilidade em água quente (\%) & 9,24 & 0,08 \\
\hline Extrativos em etanol-tolueno (\%) & 7,58 & 3,73 \\
\hline Extrativos Totais (\%) & 10,33 & 3,43 \\
\hline pH (24 horas) & 5,54 & 0,58 \\
\hline Materiais inorgânicos (\%) & 0,74 & 2,19 \\
\hline
\end{tabular}

*Densidade básica ponderada.

De acordo com a Tabela 2 pode-se verificar que a espécie apresentou densidade básica de $0,33 \mathrm{~g} / \mathrm{cm}^{3}$, sendo classificada como espécie produtora de madeira leve ou de baixa densidade. No entanto, mesmo com baixa densidade a espécie pode ser utilizada para produção de painéis aglomerados, onde Maloney (1993) afirma que espécies com até $0,55 \mathrm{~g} / \mathrm{cm}^{3}$ são adequadas para esta finalidade por atingirem uma razão de compactação entre 1,3 e 1,6, sendo esta a faixa ideal para o processo de densificação e consolidação do painel até a espessura final.

Em comparação com os resultados disponíveis em literatura, pode-se dizer que os valores médios obtidos nesta pesquisa são compatíveis com os apresentados por Pereyra et al., (2006) que encontraram valor médio de $0,337 \mathrm{~g} / \mathrm{cm}^{3}$ para árvores com idade entre 18 e 20 anos localizadas em Misiones, Ziech (2008), que obteve valores de $0,245 \mathrm{~g} / \mathrm{cm}^{3}$ a $0,364 \mathrm{~g} / \mathrm{cm}^{3}$ para árvores com 4 anos provenientes da região de Minas Gerais e por Bufalino et al., (2012) que obtiveram média de $0,320 \mathrm{~g} / \mathrm{cm}^{3}$ a partir de árvores com 18 anos de idades procedentes da região do Espírito Santo.

Em relação às propriedades químicas, verifica-se que a espécie apresenta elevado teor de componentes solúveis, onde o teor de extrativos totais foi de $10,33 \%$, sendo este valor bastante superior ao apresentado por Fengel e Wegener (1989), que descrevem intervalo de $1 \%$ a $5 \%$ para espécies pertencentes ao grupo das folhosas. No que diz respeito a influência destes componentes sobre a qualidade de colagem, pode-se dizer que um elevado teor de extrativos normalmente tende a exercer influências negativas sobre as reações entre adesivo e substrato, no entanto, há que se considerar as informações relatadas por Lima et al. (2007), que afirmam que as reações de polimerização do adesivo e as reações entre adesivos e extrativos dependem não somente da quantidade, mas também da natureza do extrativo.

Para a propriedade de $\mathrm{pH}$, observa-se que o resultado médio foi de 5,54, onde este valor apresenta-se de acordo com o intervalo de variação mencionado por Stamm (1964), e indica que a espécie não possui acidez elevada. Por outro lado, e considerando a produção de painéis aglomerados colados com resina uréica, pode-se dizer que a adição de catalisador facilita a cura da resina que ocorre entre 3,0 e 3,5 (Poblete e Zarate, 1986 citados por Poblete e Pinto, 1993), reduzindo o tempo de prensagem e, com isso, gerando maior produtividade. 
Para os componentes químicos caracterizados como materiais inorgânicos, nota-se que o valor médio foi de $0,74 \%$, o qual apresenta-se no intervalo de $0,2 \%$ a $1 \%$, mencionado por Tsoumis (1991), e bastante inferior aos relatados por Browing (1963), Sjöström (1981) e Fengel e Wegener (1989), que afirmam que em espécies de climas tropicais este componente pode atingir até $5 \%$ do peso seco. Em relação à influência deste componente sobre o processamento da madeira para produção de painéis de madeira aglomerada, pode-se dizer que a espécie não apresenta teor de materiais inorgânicos que comprometa ou gere elevado desgaste nas ferramentas de corte ou picadores.

\section{Propriedades dos painéis}

\section{Densidade aparente e razão de compactação}

$\mathrm{Na}$ tabela 3 são apresentados os valores médios obtidos para as propriedades de densidade aparente $(12 \%)$ e razão de compactação dos painéis.

TABela 3. Resultados médios da densidade aparente e razão de compactação dos painéis.

\begin{tabular}{ccc}
\hline $\begin{array}{c}\text { Tratamento } \\
D_{\text {nominal }}-T R\end{array}$ & $\begin{array}{c}\text { Densidade } \\
\left(\mathrm{g} / \mathrm{cm}^{3}\right)\end{array}$ & Razão de compactação \\
\hline $0,80-8 \%$ & $0,777 \mathrm{a}$ & $2,355 \mathrm{a}$ \\
& $(1,83)$ & $(1,83)$ \\
\hline $0,80-6 \%$ & $0,775 \mathrm{a}$ & $2,348 \mathrm{a}$ \\
& $(1,62)$ & $(1,62)$ \\
\hline $0,70-8 \%$ & $0,691 \mathrm{~b}$ & $2,094 \mathrm{~b}$ \\
& $(3,79)$ & $(3,79)$ \\
\hline $0,70-6 \%$ & $0,700 \mathrm{~b}$ & $2,121 \mathrm{~b}$ \\
& $(3,45)$ & $(3,45)$ \\
\hline $0,60-8 \%$ & $0,601 \mathrm{c}$ & $1,821 \mathrm{c}$ \\
& $(4,14)$ & $(4,14)$ \\
\hline $0,60-6 \%$ & $0,600 \mathrm{c}$ & $1,818 \mathrm{c}$ \\
& $(2,31)$ & $(2,31)$ \\
\hline
\end{tabular}

$\mathrm{D}_{\text {nominal }}$ : Densidade nominal; TR: Teor de resina; Médias seguidas de mesma letra na mesma coluna são estatisticamente iguais pelo Teste de Tukey a 95\% de probabilidade; Valores entre parênteses referem-se ao Coeficiente de Variação.

Os valores médios da densidade aparente dos painéis apresentaram valores muito próximos aos estabelecidos, onde os pequenos desvios foram observados principalmente nos tratamentos com densidade nominal mais elevada e podem ser justificados pelo fenômeno de retorno em espessura após a prensagem. Verifica-se ainda, que entre os tratamentos com mesma densidade nominal os valores médios foram muito próximos entre si, indicando que houve bom controle no experimento.

Em relação à razão de compactação, pode-se verificar que os valores médios variaram no intervalo de 1,818 a 2,355; sendo estes valores superiores aos mencionados por Kelly (1977), Moslemi (1974) e Maloney (1993), que descrevem como faixa ideal, razão de compactação entre 1,3 a 1,6. Estes elevados valores são explicados pela baixa densidade da madeira de Toona ciliata $\left(0,33 \mathrm{~g} / \mathrm{cm}^{3}\right)$ e pelo aumento da densidade nominal dos tratamentos propostos. Pesquisas com painéis aglomerados que apresentaram elevada razão de compactação foram também desenvolvidas por Iwakiri et al. (2010) e Mendes et al. (2010).

\section{Absorção de água e inchamento em espessura após 2 e 24 horas de imersão}

$\mathrm{Na}$ tabela 4 são apresentados os valores médios encontrados para a absorção de água e inchamento em espessura após 2 e 24 horas de imersão.

Para a propriedade de absorção de água os valores médios variaram de $10,89 \%$ a $16,84 \%$ e de $32,33 \%$ a $50,59 \%$ após 2 e 24 horas, respectivamente. Pode-se verificar que ocorreu o aumento da absorção com a redução da densidade nominal, onde este resultado é justificado segundo os relatos de Vital et al. (1974) que mencionam que uma maior densidade nominal proporcionam uma redução na porosidade do painel, dificultando assim a absorção de água. Nota-se também, a partir dos trata- 
mentos de mesma densidade nominal, que o aumento do teor de resina proporcionou uma ligeira redução na absorção, sendo este comportamento explicado pelo fato de que o maior teor de resina aplicado proporciona um maior recobrimento das partículas, tornando a superfície de contato menos permeável.

TABELA 4. Resultados médios da absorção de água e inchamento em espessura após 2 e 24 horas de imersão.

\begin{tabular}{ccccc}
\hline Tratamento & AA 2h & AA 24h & IE 2h & IE 24h \\
\cline { 2 - 5 }$D_{\text {nominal }}-T R$ & $(\%)$ & $(\%)$ & $(\%)$ & $(\%)$ \\
\hline $0,80-8 \%$ & $10,89 \mathrm{~d}$ & $32,33 \mathrm{c}$ & $6,23 \mathrm{a}$ & $12,84 \mathrm{~b}$ \\
& $(4,95)$ & $(5,76)$ & $(16,02)$ & $(8,89)$ \\
\hline $0,80-6 \%$ & $13,57 \mathrm{c}$ & $43,88 \mathrm{~b}$ & $6,97 \mathrm{a}$ & $17,38 \mathrm{a}$ \\
& $(9,69)$ & $(6,24)$ & $(39,28)$ & $(18,07)$ \\
\hline $0,70-8 \%$ & $14,18 \mathrm{bc}$ & $40,86 \mathrm{~b}$ & $6,22 \mathrm{a}$ & $12,74 \mathrm{~b}$ \\
& $(9,04)$ & $(8,97)$ & $(30,25)$ & $(12,64)$ \\
\hline $0,70-6 \%$ & $14,46 \mathrm{bc}$ & $43,84 \mathrm{~b}$ & $7,34 \mathrm{a}$ & $15,87 \mathrm{a}$ \\
& $(13,45)$ & $(13,95)$ & $(14,70)$ & $(10,05)$ \\
\hline $0,60-8 \%$ & $16,00 \mathrm{ab}$ & $45,26 \mathrm{~b}$ & $6,26 \mathrm{a}$ & $12,26 \mathrm{~b}$ \\
& $(9,04)$ & $(6,81)$ & $(14,05)$ & $(11,94)$ \\
\hline $0,60-6 \%$ & $16,84 \mathrm{a}$ & $50,59 \mathrm{a}$ & $6,57 \mathrm{a}$ & $15,79 \mathrm{a}$ \\
& $(13,16)$ & $(7,63)$ & $(22,96)$ & $(8,69)$ \\
\hline
\end{tabular}

$\mathrm{D}_{\text {nominal }}$ Densidade nominal; TR: Teor de resina; AA: Absorção de água; IE: Inchamento em espessura; Médias seguidas de mesma letra na mesma coluna são estatisticamente iguais pelo Teste de Tukey a 95\% de probabilidade; Valores entre parênteses referem-se ao Coeficiente de Variação.

Em relação ao inchamento em espessura, a variação entre tratamentos compreendeu valores de $6,22 \%$ a $7,34 \%$ após 2 horas de imersão, onde para este intervalo de tempo não foi constatada diferença estatística significativa. Já após 24 horas, os valores médios de inchamento variaram de $12,26 \%$ a $17,38 \%$, sendo observada diferença estatística significativa entre os tratamentos propostos.
Verifica-se, a partir dos tratamentos produzidos com diferentes densidades e com o mesmo percentual de resina $\left(0,60 \mathrm{~g} / \mathrm{cm}^{3} ; 0,70 \mathrm{~g} / \mathrm{cm}^{3} \mathrm{e} 0,80 \mathrm{~g} / \mathrm{cm}^{3}\right)$, que o aumento da densidade promoveu um ligeiro aumento no valor médio do inchamento em espessura. Este comportamento se deve ao fato de que nos painéis de maior densidade, a razão de compactação é mais elevada, ocorrendo uma maior liberação das tensões de prensagem por ocasião da imersão. Resultados similares foram também observados por Halligan (1970), onde este autor verificou que o aumento da densidade nominal gerava um aumento na propriedade de inchamento, no entanto, este aumento também não apresentava comportamento linear.

Por outro lado, analisando os diferentes níveis de resina em cada par de tratamentos com mesma densidade nominal, nota-se que o aumento do teor de resina proporcionou uma melhoria na propriedade de estabilidade, que variou de $21,87 \%$ a $31,08 \%$. Resultados semelhantes foram também encontrados por Lehmann (1974), Brito (1984), Keinert Jr e Matos (1987) e Iwakiri et al., (2000) que desenvolvendo estudos com diferentes percentuais de resina observaram redução na propriedade de inchamento.

\section{Propriedades mecânicas}

Na tabela 5 são apresentados os resultados médios para as propriedades de módulo de ruptura e módulo de elasticidade à flexão estática, tração perpendicular a superfície e resistência ao arrancamento de parafuso no topo e na superfície.

Os valores médios do módulo de ruptura e do módulo de elasticidade variaram de 13,02 MPa a 22,13 MPa e de $1891 \mathrm{MPa}$ a $2926 \mathrm{MPa}$, respectivamente, onde pode-se verificar que com o aumento da densidade nominal, tanto o MOR quanto o MOE tenderam a apresentar valores médios mais elevados. Entre tratamentos com a mesma densidade nominal e diferentes níveis de resina, também observou-se tendência de melhoria nas propriedades. Para os ensaios de tração perpendicular e resistência ao arrancamento de parafuso comportamento similar foi observado. 
TABela 5. Resultados médios das propriedades de flexão estática, tração perpendicular e resistência ao arrancamento de parafuso.

\begin{tabular}{|c|c|c|c|c|c|}
\hline \multirow{2}{*}{$\begin{array}{l}\text { Tratamento } \\
D_{\text {nominal }}-T R\end{array}$} & \multicolumn{2}{|c|}{ Flexão Estática } & \multirow{2}{*}{$\begin{array}{c}\text { Tração perpendicular } \\
\text { (MPa) }\end{array}$} & \multicolumn{2}{|c|}{ Resistência ao Arrancamento de Parafuso } \\
\hline & $\begin{array}{l}\text { MOR } \\
\text { (MPa) }\end{array}$ & $\begin{array}{l}\text { MOE } \\
\text { (MPa) }\end{array}$ & & $\begin{array}{l}\text { Topo } \\
\text { (N) }\end{array}$ & Superfície (N) \\
\hline $0,80-8 \%$ & $\begin{array}{l}22,13 \text { a } \\
(6,09)\end{array}$ & $\begin{array}{c}2926 \text { a } \\
(2,22)\end{array}$ & $\begin{array}{l}1,24 \mathrm{a} \\
(15,05)\end{array}$ & $\begin{array}{l}1856 \mathrm{a} \\
(7,43)\end{array}$ & $\begin{array}{l}1823 \text { a } \\
(10,19)\end{array}$ \\
\hline $0,80-6 \%$ & $\begin{array}{c}17,82 \text { bc } \\
(8,61)\end{array}$ & $\begin{array}{c}2435 \\
b c \\
(6,98)\end{array}$ & $\begin{array}{l}0,85 \text { b } \\
(18,54)\end{array}$ & $\begin{array}{l}1583 \text { b } \\
(11,76)\end{array}$ & $\begin{array}{c}1771 \mathrm{ab} \\
(11,82)\end{array}$ \\
\hline $0,70-8 \%$ & $\begin{array}{c}19,13 \mathrm{ab} \\
(9,76)\end{array}$ & $\begin{array}{c}2684 \\
a b \\
(3,73)\end{array}$ & $\begin{array}{l}0,89 \mathrm{~b} \\
(30,94)\end{array}$ & $\begin{array}{c}1456 \text { bc } \\
(9,66)\end{array}$ & $\begin{array}{l}1586 \text { b } \\
(10,95)\end{array}$ \\
\hline $0,70-6 \%$ & $\begin{array}{c}18,50 \mathrm{bc} \\
(8,12)\end{array}$ & $\begin{array}{c}2485 \\
b c \\
(10,53)\end{array}$ & $\begin{array}{c}0,87 \text { b } \\
(20,48)\end{array}$ & $\begin{array}{l}1304 \mathrm{c} \\
(11,28)\end{array}$ & $\begin{array}{l}1543 \text { b } \\
(11,80)\end{array}$ \\
\hline $0,60-8 \%$ & $\begin{array}{l}15,61 \mathrm{~cd} \\
(17,99)\end{array}$ & $\begin{array}{l}2356 \mathrm{c} \\
(12,66)\end{array}$ & $\begin{array}{l}0,81 \mathrm{~b} \\
(22,25)\end{array}$ & $\begin{array}{l}1069 d \\
(15,03)\end{array}$ & $\begin{array}{l}1187 \mathrm{c} \\
(12,92)\end{array}$ \\
\hline $0,60-6 \%$ & $\begin{array}{l}13,02 \mathrm{~d} \\
(9,36)\end{array}$ & $\begin{array}{l}1891 \mathrm{~d} \\
(5,33)\end{array}$ & $\begin{array}{l}0,74 \text { b } \\
(17,39)\end{array}$ & $\begin{array}{l}1008 d \\
(8,40)\end{array}$ & $\begin{array}{l}1151 \mathrm{c} \\
(8,35)\end{array}$ \\
\hline
\end{tabular}

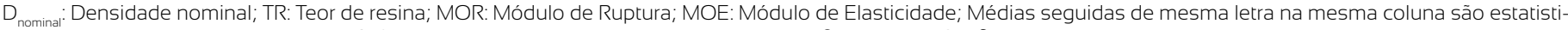
camente iguais pelo Teste de Tukey a 95\% de probabilidade; Valores entre parênteses referem-se ao Coeficiente de Variação.

De maneira geral, pode-se verificar que todos os tratamentos atenderam os requisitos mínimos estabelecidos pela norma EN 312:2003 (CEN, 2003), de 13 MPa para o Módulo de ruptura, $1600 \mathrm{MPa}$ para Módulo de elasticidade, 0,35 MPa para tração perpendicular e $800 \mathrm{~N}$ e $1020 \mathrm{~N}$ para arrancamento de parafuso no topo e na superfície, respectivamente, sendo tecnicamente viável a utilização da espécie para a geração de produtos com diferentes densificações e resistências.

Em comparação com os resultados obtidos para esta mesma espécie, nota-se que Sá (2009) avaliando as propriedades de painéis aglomerados com densidade nominal de 0,70 e teor de resina de $9 \%$ (parâmetros de prensagem similares ao utilizados neste trabalho) obteve para flexão estática, módulo de ruptura e de elasticidade de 17,21 MPa e 1302 MPa respectivamente, onde estes resultados são inferiores ao deste trabalho para a mesma classe de densidade nominal, mesmo com maior teor de resina. Já para a propriedade de tração perpendicular a autora encontrou valor médio de 1,29 $\mathrm{MPa}$, sendo este bastante superior os resultado da presente pesquisa.

Por outro lado, buscando a maximização deste recurso florestal e conseqüentemente da quantidade de resina utilizada para a consolidação dos painéis, nota-se que o trata- 
mento com menor quantidade de matéria prima empregada $\left(0,60 \mathrm{~g} / \mathrm{cm}^{3}\right.$ e $6 \%$ de resina) atende o valor mínimo de resistência, o que indica um resultado promissor. Em termos de quantificação de matéria prima no processo produtivo, verifica-se que ocorre uma redução de até $23,73 \%$ na quantidade de partículas ( $3 \%$ de umidade), até $42,80 \%$ nas quantidades de resina e catalisador e até $25 \%$ na quantidade de parafina.

\section{CONCLUSÕES}

De acordo com os resultados obtidos as seguintes conclusões podem ser apresentadas:

Todos os tratamentos indicaram viabilidade técnica para a produção de painéis aglomerados, atendendo os requisitos mínimos exigidos pelas normas de referência, e gerando produtos com diferentes classes de qualidade.

Em relação à estabilidade dimensional, pode-se dizer que o teor de resina exerceu maior influência sobre a absorção de água e o inchamento em espessura do que a densidade nominal.

A produção de painéis aglomerados de Toona ciliata com densidade nominal de $0,60 \mathrm{~g} / \mathrm{cm}^{3}$ e $6 \%$ de resina, além de atender os requisitos normativos implica numa redução até $23,73 \%$ na quantidade de partículas, até $42,80 \%$ nas quantidades de resina e catalisador, e até $25 \%$ na quantidade de parafina, quando comparado com os demais tratamentos.

\section{RECONHECIMENTOS}

Ao CNPq, à Battistella Florestal e à Arauco do Brasil pelo suporte a esta pesquisa.

\section{REFERÊNCIAS}

Abipa (Associação Brasileira da Indústria de Painéis de Madeira). 2012. Disponível em: <http://www.abipa.org. br/meioambiente.php> Acesso em 05/08/2012.

ABNT (Associação Brasileira de Normas Técnicas). 2006a. NBR 14810 - 2 - Chapas de madeira aglomerada - Parte 2 - Requisitos.

Associação Brasileira de Normas Técnicas - ABNT. 2006b. NBR 14810 - 3 - Chapas de madeira aglomerada - Parte 3 Métodos de Ensaio.
Boletim Florestal. 2008. Cedro australiano: Valorização de espécies nobres. Boletim Florestal: Informativo Florestal do Norte Pioneiro. 7(2) :2-4.

Brito, E.O.A. 1984. Viabilidade de utilização de espécies de Pinus para a produção de chapas de composição estruturais "waferboards". Dissertação (Mestrado em Ciências Florestais), Setor de Ciências Agrárias, Universidade Federal do Paraná, Curitiba, 102 p.

Browing, B.L. 1963. The Chemistry of wood. John Wiley \& Sons, New York 689p.

Bufalino, L., T.P. Protásio, A.A.S. César, V.A. Sá, e L.M. Mendes. 2012. Modelagem das propriedades físicas e mecânicas em painéis aglomerados de cedro australiano. Floresta e ambiente 19(2):243-249.

COPAnT (Comissão Panamericana de Normas Técnicas). 1972. COPANT 461. Determinación del peso especifico aparente.

Dordel, J. e S. Simard. 2009. Nurse-tree effects on Autralian Red Cedar (Toona ciliata): a comparison of there nurse species. In: 6th North American Forest Ecology Workshop, Proocedings.

CEN (European Committee for Standardization). 2002. EN 310. Determination of modulus of elasticity in bending and of bending strength. Versão portuguesa.

CEN (European Committee for Standardization). 2003. EN 312. Particleboards - Specifications. Versão em Inglês.

CEN (European Committee for Standardization). 2002. EN 317. Determination of swelling in thickness after immersion in water. Versão em Inglês.

CEN (European Committee for Standardization). 2002. EN 319. Determinação da resistência à tração perpendicular às faces da placa. Versão Portuguesa.

CEN (European Committee for Standardization). EN 323. Determinação da massa volúmica. Versão Portuguesa.

Fengel, D. e G. Wegener, 1989. Wood Chemistry ultrastructure reactions. Waster \& Grugter. Berlin. 612 p.

Halligan, A. F. 1970. A review of tickness swelling in particleboard. Wood science and technology 4:301-312.

Herwitz, S.R., R.E. Slye e S.M. Turton. 1998. Redifining the ecological niche of a tropical rain forest canopy tree species using airborne imagery: long-term crown dynamics 
of Toona ciliata. Journal of Tropical Ecology 4:683703.

Iwakiri, S. 2005. Painéis de madeira reconstituída. Fupef. Curitiba. 247 p.

Iwakiri, S., A.B. Cunha, C.E. Albuquerque, E. Gorniak e L.M. Mendes. 2000. Resíduos de serrarias na produção de painéis de madeira aglomerada de eucalipto. Scientia Agrária $1(1-2): 23-28$.

Iwakiri, S., F. Zeller, J.A. Pinto, M.G.L. Ramirez, M.M. Souza e R. Seixas. 2010. Avaliação do potencial de utilização da madeira de Schizolobium amazonicum "Paricá" e Cecropia hololeuca "Embauba"para a produção de painéis aglomerados. Acta Amazonica 40(2):303-308.

Keinert JR. S. e J.L.M. Matos. 1987. Utilização de Pinus pinaster para fabricação de chapas de partículas. Floresta 17(12):113-120.

Kelly, M.W.A. 1977. Critical literature review of relationships between processing parameters and physical properties of particleboards. U.S. For. Prod. Lab. General Technical. Madison. 66 p.

Keenan R.; Lamb. D.; Woldring, O.; Irvine, T.; Jensen, R. 1997. Restoration of plant diversity beneath tropical tree plantations in Northern Australia. Forest Ecology and Management 99:117-131.

Lamprecht, H. 1990. Silvicultura nos trópicos: ecossistemas florestais e respectivas espécies arbóreas - possibilidades e métodos de aproveitamento sustentado. Rossdorf: TZ Verl.- Ges. 343 p.

Lehmann, W.F. 1974. Properties of structural particleboards. Forest Products Journal 24(1):19-26.

Lima, C.K. P., F.A. Mori, L.M. Mendes e A.C.O. Carneiro. 2007. Características anatômicas e química da madeira de clones de Eucalyptus e sua influência na colagem. Cerne 13(2):123-129.

Maloney, T.M. 1993. Modern particleboard and dry-process fiberboard manufacturing. 2a. ed. Miller Freeman. São Francisco. 689 p.

Marra, A.A. 1992. Technology of Wood bonding: principles and practice. Van Nostrand Reinhold. New York. 453 p.

Mendes, R.F., L.M. Mendes, R.A.S. Abranches, R.C. Santos e J.B. Guimarães Junior. 2010. Painéis aglomerados pro- duzidos com bagaço de cana em associação com madeira de eucalipto. Scientia Forestalis 38(86):285-295.

Moslemi, A.A. 1974. Particleboard. Southern Illinois University Press. London. 245p.

Paiva, Y.G., G.S. Mendonça, K.R. Silva, M.E. Nappo, R.A. Cecílio e J.E.M. Pezzopane. 2007. Zoneamento agroecológico de pequena escala para Toona ciliata, Eucalyptus grandis e Eucalyptus urophylla na Bacia Hidrográfica do Rio Itapemirim - ES, utilizando dados SRTM. In: XIII Simpósio Brasileiro de Sensoriamento Remoto, Florianópolis, Anais, INPE. 1785-1792.

Pereyra, O.; Suirezs, T.M.; Pitsch, C.; Báez, R. 2006. Estudio de las propiedades fisico-mecánicas y comportamiento en procesos industriales de la madera de kiri, grevillea, paraíso y toona. Floresta 36(2): 213-223.

Poblete, H.W. e A.S. Pinto. 1993. Avances sobre el efecto del catalizador em el fraguado de ureaformaldeido em tableros de tepa. Bosque 14(1):55-61.

Sá, V.A. 2009. Potencial da madeira de Cedro australiano (Toona ciliata M. Roem var. australis) na manufatura de produtos de maior valor agregado. Dissertação (Mestrado em Ciência e Tecnologia da Madeira) - Universidade Federal de Lavras, Lavras. 82 p.

Sjöström. E. 1981. Wood Chemistry fundamentals and applications. Academic Press. New York. 223p.

Stamm, A.J. 1964. Wood and cellulose science. The Ronald Press Company. New York. 549p.

TAPPI (Technical Association of the Pulp and Paper Industry). 1997a. TAPPI 264. Preparation of wood for chemical analysis. Atlanta.

TAPPI (Technical Association of the Pulp and Paper Industry). 1997b. TAPPI 204. Solvent extractives of wood and pulp. Atlanta.

TAPPI (Technical Association of the Pulp and Paper Industry). 1999a. TAPPI 207. Water solubility of wood and pulp. Atlanta.

TAPPI (Technical Association of the Pulp and Paper Industry). 1999b. TAPPI 280. Acetone extractives of wood and pulp. Atlanta.

TAPPI (Technical Association of the Pulp and Paper Industry). 2002a. TAPPI 211. Ash in wood, pulp, paper and paperboard: combustion at $525^{\circ} \mathrm{C}$. Atlanta. 
TAPPI (Technical Association of the Pulp and Paper Industry). 2002b. TAPPI 252. pH and electrical conductivity of hot water extracts of pulp, paper, and paperboard. Atlanta.

Tomaselli, I. 1999. Tendências de mudanças na Indústria de Painéis. Revista da Madeira 8(43): 36-40.

Trianoski, R. 2010. Avaliação do potencial de espécies florestais alternativas de rápido crescimento para produção de painéis de madeira aglomerada. Dissertação (Mestrado em Ciências Florestais) - Universidade Federal do Paraná, Curitiba. 262p.

Tsoumis, G. 1991. Science and technology of wood: structure, properties and utilization. New York, $494 \mathrm{p}$.

Vital, B.R., W.F. Lehmann e R.S. Boone. 1974. How species and board densities affect properties of exotic hardwood particleboards. Forest Products Journal 24(12):37-45.
Ziech, R.Q.S. 2008. Características tecnológicas da madeira de cedro australiano (Toona ciliata M. Roem) produzida no sul do Estado de Minas Gerais. Dissertação (Mestrado em Ciência e Tecnologia da Madeira) Universidade Federal de Lavras, Lavras. 91p.

Manuscrito recibido el 2 de octubre de 2012 Aceptado el 4 de agosto de 2014.

Este documento se debe citar como:

Trianoski, R., S. Iwakiri, J. L. M. Matos. Avaliação de painéis aglomerados de Toona ciliata produzidos com diferentes densidades e teores de resina. Madera y Bosques 20(3):49-58. 